EPJ Web of Conferences 116, 06002 (2016)

DOI: $10.1051 /$ epjconf/201611606002

(C) Owned by the authors, published by EDP Sciences, 2016

\title{
Aspects of the optical system relevant for the KM3NeT timing calibration
}

\author{
Gerard Kieft on behalf of the KM3NeT Collaboration \\ Nikhef, Science Park 105, 1098 XG Amsterdam, The Netherlands
}

\begin{abstract}
KM3NeT is a future research infrastructure in the Mediterranean Sea housing the large Cherenkov telescope arrays of optical modules for neutrino detection. The detector control and data transmission system is based on fibre optical technology. For timing calibration of the detector signals the optical system is used to send and fan-out an onshore clock signal, derived from a GPS receiver, to all optical modules in the deep sea. The optical modules use this clock signal to time stamp the light pulses detected by the photomultipliers inside the modules. The delay time between the GPS clock on shore and the clock in each optical module is measured with sub-nanosecond precision using a White Rabbit based timing calibration system. The aspects of the optical system relevant for the timing calibration and the quantification of their effect will be presented.
\end{abstract}

\section{Introduction}

The final configuration of the arrays of optical modules of the KM3NeT neutrino telescope being installed at the bottom of the Mediterranean Sea will comprise a total instrumented volume of several cubic kilometres to detect Cherenkov light from traversing neutrino-induced leptons [1]. During the first construction phase 31 detection units (DU) will be installed configured as long vertical lines each containing 18 Digital Optical Modules (DOM) and each DOM containing 31 photomultipliers [2]. These DUs will be deployed at two locations in the Mediterranean at depth of $2500 \mathrm{~m}$ (KM3NeT-Fr) and $3500 \mathrm{~m}$ (KM3NeT-It) and connected to seabed networks of electro-optical cables and junction boxes. Vertical electro-optical cables (VEOCs) run the full length of a DU. At the anchor of each DU a dedicated base-module is mounted for connection of the VEOC to the seabed infrastructure. Main electro-optical cables (MEOCs) connect the seabed infrastructures with the shore station at distances between $40 \mathrm{~km}$ (KM3NeT-Fr) and $100 \mathrm{~km}$ (KM3NeT-It). For communication between the shore station and each DOM the implemented optical system consists of a single optical broadcast from shore to all the DUs and a DWDM based optical paths over several fibres for transport of the data from the DUs to shore. For the broadcast one wavelength is used to distribute a GPS derived system clock and the slow control data to each DOM. To send data to shore every base-module and each DOM use a dedicated wavelength in a band of at maximum 72 wavelengths per fibre.

Temperature, pressure and wavelength have an effect on the propagation delay of optical pulses over the fibres. The KM3NeT timing calibration depends on the measurement of the delay between the system clock on shore and all the DU-base modules and DOMs. After an introduction on the principle

This is an Open Access article distributed under the terms of the Creative Commons Attribution License 4.0, which permits unrestricted use, distribution, and reproduction in any medium, provided the original work is properly cited. 


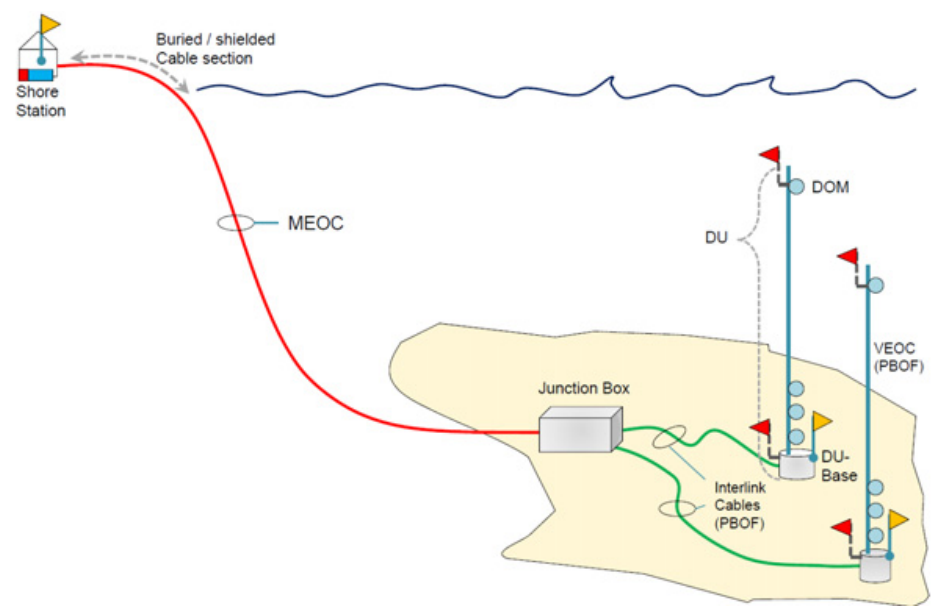

Figure 1. Schematic view of the KM3NeT optical scheme.

of timing calibration in Sect. 2, measurements of the wavelength-dependent propagation delays of the optical fibres in the MEOC are reported in Sect. 3. The effects of pressure and temperature on the propagation delays over the optical fibres in the VEOC of a DU (Fig. 1) are described in Sects. 4 and 5.

\section{Principle of the timing calibration}

The aim of the timing calibration system is to measure for each DOM the time delay between a GPS derived system clock on shore and the arrival of a timed laser pulse on a reference PMT in each DOM. This delay is measured in 2 steps:

1. White Rabbit (WR) protocol and hardware [3] is used to measure the RTT (Round Trip Time) between the system clock on shore and the clock on the CLB (Central Logic Board) in a DUbase module (between the yellow flags in Fig. 1). When the difference in the propagation time (asymmetry) from shore to DU-base and from DU-base to shore is known, the delay between the system clock on shore and the clock in the DU-base can be derived from the RTT.

2. The delay in the VEOC between the DU-base and each DOM in a DU is measured before deployment during a darkroom test on shore (between the red flags in Fig. 1).

\section{Wavelength-dependent propagation delay}

The propagation delay of optical pulses over a fibre is determined by the Group Refractive Index (GRI) which depends on the wavelength. For the broadcast signal from shore to a DU-base a different wavelength is used than for the Detector Control (DC) signal from a DU-base back to shore (Fig. 2). Due to the different propagation delay times, the measured RTT between the shore and a DU-base is asymmetric. To derive the propagation delay from shore to a DU-base from the RTT, the asymmetry must be known. Therefore, the wavelength dependencies of the propagation delay of the optical fibres in the MEOCs are measured. For an accurate calculation of the propagation delay of optical signals over fibres, the Group Velocity (GV) at the wavelength of interest must be known. The GV for optical signals in the MEOCs are measured for a set of 8 wavelengths.

Using WR the RTT in a stand-alone reference set-up is measured (Fig. 3a). This is followed by a RTT measurement of the same reference set-up but now including a MEOC fibre (Fig. 3b). By subtracting the 


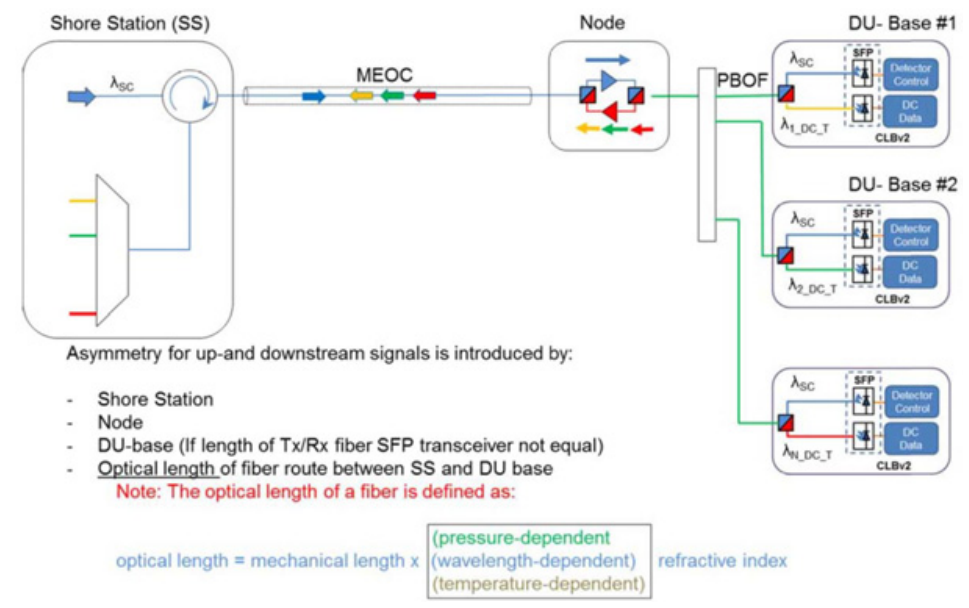

Figure 2. Wavelengths between shore station and DU-base.

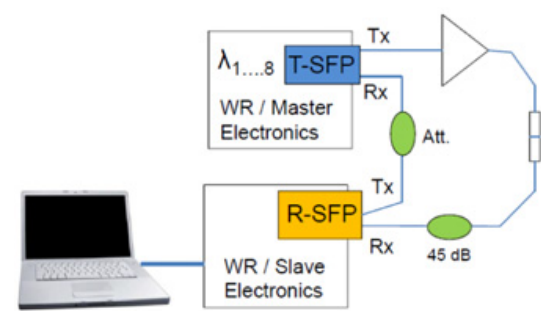

Figure 3a. Reference set-up.

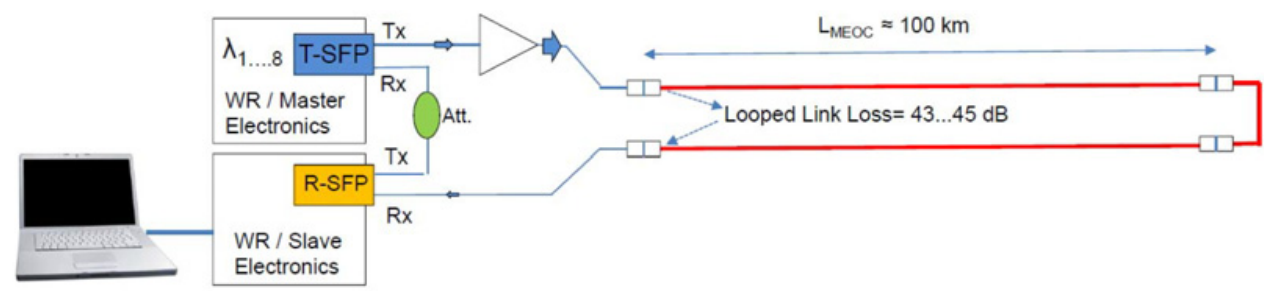

Figure 3b. Reference set-up including the MEOC fibre.

reference RTT from the second RTT, the propagation delay for the specific wavelength in the MEOC fibre can be calculated. For the MEOC at KM3NeT-Fr six fibres, to be used for the SC/DC optical signals, were measured prior to deployment of the MEOC. Since at KM3NeT-It the MEOC was already deployed, the measurement could only be performed on three sets of two fibres in series. Each set of fibres was looped-back in the offshore cable termination.

Figure 4 shows the Relative Group Delay (RGD, the difference in GV) measured for the MEOC of KM3NeT-It. The results represent the average values for 3 different loops of 2 fibres in series. For this MEOC, using a wavelength range from $1530-1544 \mathrm{~nm}$, the RGD for a fibre of $\sim 96.76 \mathrm{~km}$ length ranges between ca. $5.2 \mathrm{~ns}$ and $5.4 \mathrm{~ns}$. Figure 5 shows the RGD measured for the MEOC of KM3NeT-Fr. The results represent the values for 6 single fibres. For this MEOC, using a wavelength range from $1530-1552 \mathrm{~nm}$, the RGD for a fibre of $\sim 62 \mathrm{~km}$ length ranges between ca. $7.2 \mathrm{~ns}$ and $8.0 \mathrm{~ns}$. 


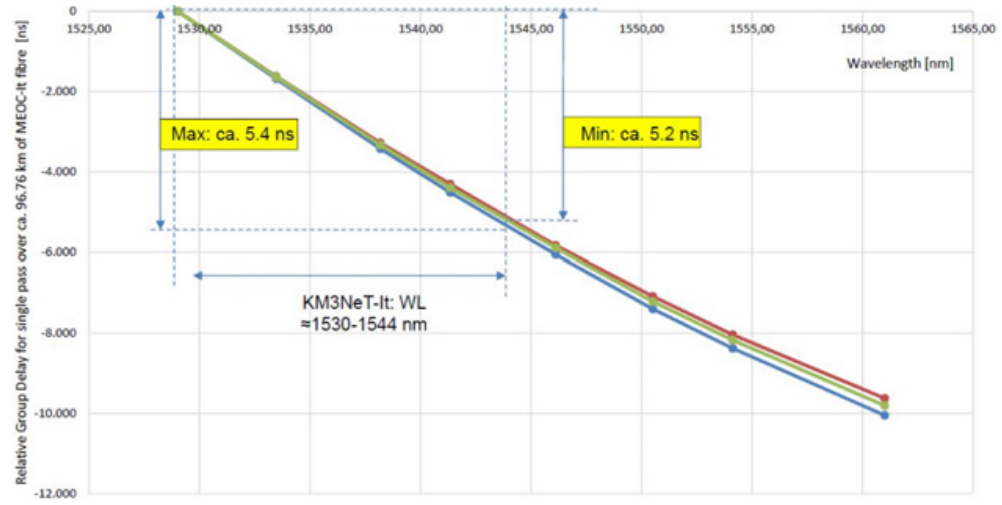

Figure 4. RGD measured for 3 fibre loops in MEOC-Italy.

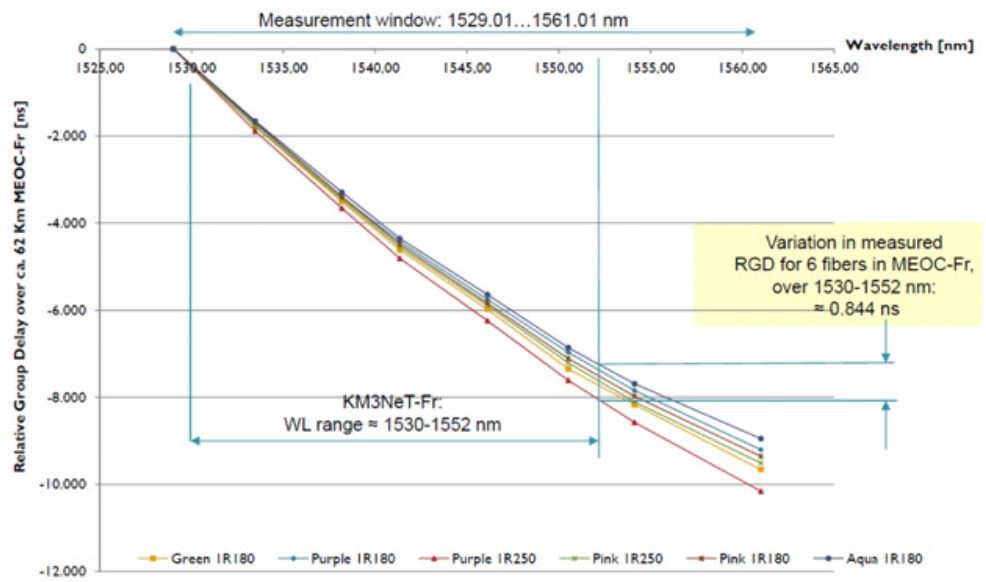

Figure 5. RGD measured for 6 fibres in MEOC-France.

For accurate calculation of the GV for specific wavelengths, all RTT measurements were fitted to a $2^{\text {nd }}$ order (quadratic) Sellmeier function which appeared to give the best fit. The fit results showed regression coefficients ranging between $\mathrm{r}^{2}=0.9998$ and $\mathrm{r}^{2}=1.0$.

\section{Effect of temperature on propagation delay}

The timing in a VEOC of a DU is calibrated in a darkroom under "Room Temperature" conditions (e.g. $23^{\circ} \mathrm{C}$ ). After deployment, the BendBrightXS fibres in VEOC of the DU will be operated in sea water of $\sim 13^{\circ} \mathrm{C}$. This temperature change will affect the propagation delay of optical pulses in the VEOC fibres. In literature, for standard SMF28 fibre with an UV-acrylate primary coating, an effect of ca. 35 till $38 \mathrm{ps} / \mathrm{km} .{ }^{\circ} \mathrm{C}$ is reported [4]. In a climate chamber the effect of temperature on the propagation delay for three spools of $4.4 \mathrm{~km}$ BendBrightXS UV-acrylate primary coated fibre ${ }^{1}$ was measured for different wavelengths. To avoid errors due to thermal expansion of the drum material, each fibre was strain-free spooled on a half-circular drum and thus partially suspended in air. The effect of the temperature on

${ }^{1}$ Provided for free by the company Prysmian, Eindhoven, The Netherlands. 


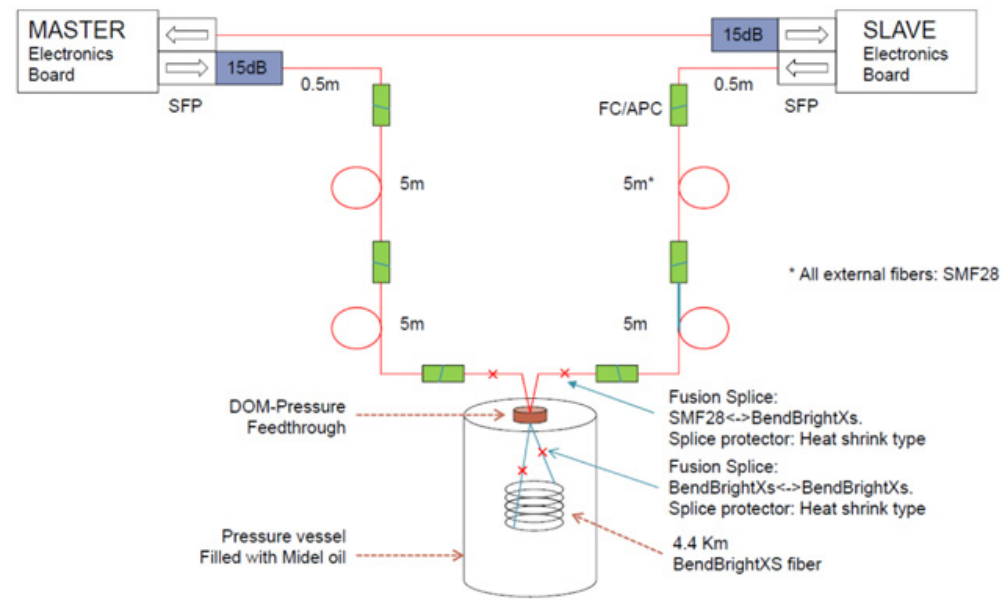

Figure 6. Set-up to measure RTT during pressure test.

the propagation delay for BendBrightXS fibre is measured as $36.7 \mathrm{ps} / \mathrm{km} .{ }^{\circ} \mathrm{C}$ and does not significantly depend on the wavelength. Due to a temperature change of ca. $-10^{\circ} \mathrm{C}$ for a DU after deployment, the change in propagation delay between the DU-base and the highest DOM (spanning $\sim 750 \mathrm{~m}$ ) is: $-10 * 0.75 * 36.7=-0.275 \mathrm{~ns}$.

\section{Effect of pressure on propagation delay}

The VEOC of the DU is calibrated under atmospheric pressure. After deployment of the DU, the BendBrightXS fibres in the VEOC will be operated at a pressure between 25 and $35 \mathrm{MPa}$ (250 and 300 bar) depending on the deployment site. This pressure change will affect the propagation of optical pulses running over the VEOC fibres. The effect of pressure on the same three spools of $4.4 \mathrm{~km}$ BendBrightXS fibre was measured in a pressure vessel. The variation of the optical attenuation was measured over a pressure range between 0.1 and $60 \mathrm{MPa}$. Within an accuracy of $0.02 \mathrm{~dB}$ no changes in optical attenuation were observed.

Using the WR protocol to measure the RTT at $1530.33 \mathrm{~nm}$, the pressure-dependent propagation delay of the three fibre spools is determined to be between -1.58 and $-1.86 \mathrm{ps} / \mathrm{bar} . \mathrm{km}$. In literature for standard SMF28 fibre an effect of ca. $-3.4 \mathrm{ps} / \mathrm{bar} . \mathrm{km}$ is reported [5]. The effect of pressure on the propagation delay of BendBrightXS fibre is about half the value as reported for SMF28 fibre. This is assumed to be due to the different material properties of the BendBrightXS fibre. At a deployment depth of $3500 \mathrm{~m}$ the average pressure over the $\sim 750 \mathrm{~m}$ length of the VEOC fibre for the highest DOM is $31.25 \mathrm{MPa}$ (312.5 bar). Due to the pressure change after deployment, the change in the propagation delay after deployment with respect to that measured on-shore during the DU calibration is: $-1.86 * 312.5 * 0.75=-0.436 \mathrm{~ns}$.

\section{Summary and conclusions}

For fibres in the MEOCs of the KM3NeT deep sea infrastructures the dependency of the propagation delay as function of the wavelength has been measured for 8 wavelengths. The resulting data have been fitted to a $2^{\text {nd }}$ order Sellmeier function. Using this function the asymmetry in the RTT between shore and offshore due to different wavelengths in the downstream and upstream optical paths, could be calculated. The effect of temperature and pressure changes on the propagation delay in the BendBrightXS fibres 


\section{EPJ Web of Conferences}

Table 1. Condensed overview of optical effects on the timing calibration.

\begin{tabular}{|c|c|c|c|c|c|}
\hline Component & $\lambda$ & $\mathrm{T}$ & $\mathrm{P}$ & $\begin{array}{l}\text { Other } \\
\text { effects }\end{array}$ & Remarks \\
\hline $\begin{array}{l}\text { MEOC fibres } \\
(50-100 \mathrm{~km})\end{array}$ & 0 & O & 0 & & $\begin{array}{l}\text { "Temperature interval: between sea floor and shore. } \\
\text { "Fibres in MEOC in stainless steel tube, with pressure seal. }\end{array}$ \\
\hline $\begin{array}{l}\text { Interlink fibres } \\
\qquad(\approx 1 \mathrm{~km})\end{array}$ & O & O & O & & $\begin{array}{l}\text { * Fibres in (horizontal) PBOF cables (Interlink cables) are exposed to constant hydrostatic } \\
\text { pressure. }\end{array}$ \\
\hline VEOC - fibres & $\odot$ & ○ & O & $\begin{array}{l}\text { Strain-induced } \\
\text { effects in } \\
\text { VEOC-fibres }\end{array}$ & $\begin{array}{l}\text { - Fibres in VEOC are exposed to variable hydrostatic pressure and (assumed to be minor) vertical } \\
\text { temperature gradients of sea water. } \\
\text { "VEOC delay times are calibrated in the lab. After deployment the VEOC is exposed to a lower } \\
\text { temperature and higher pressure and assumed not to have a significant change of the fibre strain. }\end{array}$ \\
\hline $\begin{array}{l}\text { EDFA's } \\
\text { in containers }\end{array}$ & O & O & O & $\begin{array}{l}\text { EDFA Pump } \\
\text { power settings }\end{array}$ & $\begin{array}{l}\text { "Erbium Doped fibres show generally a higher (also pump power-dependent) Chromatic } \\
\text { Dispersion than } 1550 \mathrm{~nm} \text {-Dispersion Shifted fibres, but are only } 10-40 \mathrm{~m} \text { in length. This effect will } \\
\text { be negligible. }\end{array}$ \\
\hline $\begin{array}{l}\text { Passive components } \\
\text { in containers }\end{array}$ & O & 0 & O & & $\begin{array}{l}\text { * Temperature inside containers will be higher than sea water temperature but propagation times } \\
\text { are relatively very small. }\end{array}$ \\
\hline Transceivers & O & O & O & $\begin{array}{l}\text { Input power- } \\
\text { dependent time } \\
\text { delay }\end{array}$ & $\begin{array}{l}\text { * Internal time delay of a PIN-based SFP receiver varies with ca. } 225 \mathrm{ps} \text { for a } 20 \mathrm{~dB} \text { change in } \\
\text { received optical power. Internal time delay of an APD-based SFP receiver varies }<25 \text { ps over } 20 \\
\text { dB range of received optical power. }\end{array}$ \\
\hline
\end{tabular}

of the VEOC has been measured. The resulting temperature and pressure coefficients will be used to calculate after the deployment of a DU in the deep sea, for each DOM in the DU the change of the propagation delay as measured on shore during the darkroom calibration. A condensed overview of the optical effects on the KM3Net timing calibration is shown in Table 1.

This work is part of the research programme of the 'Stichting voor Fundamenteel Onderzoek der Materie (FOM)', which is financially supported by the 'Nederlandse Organisatie voor Wetenschappelijk Onderzoek (NWO)'. The research leading to these results has received funding from the European Community's Sixth Framework Programme under contract n ${ }^{\circ} 011937$ and the Seventh Framework Programme under grant agreement n 212525.

\section{References}

[1] KM3NeT, Technical Design Report for a Deep-Sea Research Infrastructure in the Mediterranean Sea Incorporating a Very Large Volume Neutrino Telescope, ISBN 978-90-6488-031-5 (April 2008)

[2] P. Kooijman, et al., Nuclear Instruments and Methods in Physics Research, Section A 626-627 (2011) S139

[3] White Rabbit Specification v2.0 (July 2011) http://www . ohwr . org/documents/160

[4] L.G. Cohen and J.W. Flemming: Effect of Temperature on Transmission in Lightguides, The Bell System Technical Journal, 58, No.4, April 1979

[5] D. Monnier-Bourdin, Système de synchronization picoseconde et métrologie associée, Application au laser megajoule, Thèse de doctorat de l'Université Paris, 1998 